OPEN ACCESS

Edited by:

Serge Nataf,

Université Claude Bernard

Lyon 1, France

Reviewed by:

Angela Wahl,

University of North Carolina at

Chapel Hill, United States

Dong Sung An,

University of California,

Los Angeles, United States

*Correspondence:

Qingsheng Li

qli@unl.edu

Santhi Gorantla

sgorantla@unmc.edu

Specialty section:

This article was submitted to

Multiple Sclerosis

and Neuroimmunology,

a section of the journal

Frontiers in Immunology

Received: 25 February 2021

Accepted: 26 April 2021

Published: 21 May 2021

Citation:

Zhang J, Lohani SC, Cheng Y,

Wang T, Guo L, Kim W-K,

Gorantla S and Li Q (2021)

Human Microglia Extensively Reconstitute in Humanized-BLT

Mice With Human Interleukin-34

Transgene and Support

HIV-1 Brain Infection.

Front. Immunol. 12:672415. doi: 10.3389/fimmu.2021.672415

\section{Human Microglia Extensively Reconstitute in Humanized-BLT Mice With Human Interleukin-34 Transgene and Support HIV-1 Brain Infection}

\author{
Jianshui Zhang ${ }^{1,2}$, Saroj Chandra Lohani ${ }^{1,2}$, Yilun Cheng ${ }^{1,2}$, Tao Wang ${ }^{1,2}$, Lili Guo ${ }^{3}$, \\ Woong-Ki Kim ${ }^{4}$, Santhi Gorantla ${ }^{3 *}$ and Qingsheng $\mathrm{Li}^{1,2^{*}}$ \\ ${ }^{1}$ School of Biological Sciences, University of Nebraska-Lincoln, NE, United States, ${ }^{2}$ Nebraska Center for Virology, University \\ of Nebraska-Lincoln, NE, United States, ${ }^{3}$ Department of Pharmacology and Experimental Neuroscience, University of \\ Nebraska Medical Center, Omaha, NE, United States, ${ }^{4}$ Department of Microbiology and Molecular Cell Biology, Eastern \\ Virginia Medical School, Norfolk, VA, United States
}

Humanized bone marrow-liver-thymic (hu-BLT) mice develop a functional immune system in periphery, nevertheless, have a limited reconstitution of human myeloid cells, especially microglia, in CNS. Further, whether bone marrow derived hematopoietic stem and progenitor cells (HSPCs) can enter the brain and differentiate into microglia in adults remains controversial. To close these gaps, in this study we unambiguously demonstrated that human microglia in CNS were extensively reconstituted in adult NOG mice with human interleukin-34 transgene (hlL34 Tg) from circulating CD34+ HSPCs, nonetheless not in hu-BLT NOG mice, providing strong evidence that human CD34+ HSPCs can enter adult brain and differentiate into microglia in CNS in the presence of hlL34. Further, the human microglia in the CNS of hu-BLT-hIL34 NOG mice robustly supported HIV-1 infection reenforcing the notion that microglia are the most important target cells of HIV-1 in CNS and demonstrating its great potential as an in vivo model for studying HIV-1 pathogenesis and evaluating curative therapeutics in both periphery and CNS compartments.

Keywords: microglia, interleukin-34, NOG mice, Hu-BLT mice, HIV-1

\section{INTRODUCTION}

Microglia, the resident macrophages in the central nervous system (CNS), are the key resident immune cells to maintain neuronal homeostasis, defend against infections, and are associated with the pathogenesis of many neurodegenerative diseases (1-3). The ontology of adult brain microglia has been debated for a long time. The consensual view to date is that microglia in CNS is the seeding results of primitive hematomyeloid precursor cells from yolk sac and aorta-gonad-mesonephros region in early embryo life and proliferation in situ thereafter (4-8). However, multiple studies also showed that bone marrow derived cells can enter the brain and differentiate into microglia in adults $(9-11)$. 
Humanized mice (hu-mice) with a human immune system have been extensively used in investigating the ontology of immune cells, immunopathogenesis of human specific pathogens, and evaluating therapeutics as preclinical small animal models (12-15). Hu-mice generated by engrafting human CD34+ hematopoietic stem and progenitor cells (HSPCs) in neonatal life can reconstitute macrophages in the meninges and perivascular spaces, but rarely in the parenchyma of brain $(16,17)$. Similarly, humanized bone marrow-liverthymic (hu-BLT) mice engrafted with human fetal liver and thymic tissues and HSPCs at adults develop a functional immune system in periphery but have a limited reconstitution of human myeloid cells, especially microglia, in CNS (18). We previously demonstrated that human interleukin-34 transgenic (hIL34-Tg) NOG mice engrafted intrahepatically with CD34+ HSPCs at birth significantly reconstituted microglial-like cells in the CNS (19). However, it remained unknown whether adult hIL34-Tg NOG mice could also reconstitute human microglia in CNS until this study. The hu-BLT mice are the best hu-mice in terms of human immune reconstitution, as they are engrafted with human fetal thymic tissues in addition to human liver tissues and liver derived CD34+ HSPCs where human T cells can receive differentiation and selection education in human thymic tissues $(20,21)$. This study has been poised to address two questions using the hu-BLT hIL34-Tg NOG (hu-BLT-hIL34) mouse model. First, we wanted to investigate whether human parenchymal microglia in the CNS could be reconstituted in adult hIL34 Tg mice from circulating myeloid precursor cells derived from CD34+ HSPCs. This is a fundamental question regarding the origin of human microglia in CNS at adults. The second question was to test the susceptibility of the reconstituted human microglia to HIV-1 infection.

Using this unique system and by comparing two types of huBLT mice with and without hIL34 Tg received the same human donor tissues, we unambiguously demonstrated that human microglia in CNS can be extensively reconstituted in adult hIL34 Tg NOG mice but no in NOG mice, which provides strong evidence that human CD34+ HSPCs can enter adult brain and differentiate into microglia in the CNS in the presence of hIL34. Further, the human microglia in the CNS of hu-BLThIL34 mice are susceptible to HIV-1 infection, which reenforced the notion that microglia are the most important target cells of HIV-1 in CNS and demonstrated its great potential as an in vivo model for studying HIV-1 pathogenesis and evaluating curative therapeutics in both periphery and CNS compartments.

\section{METHOD}

\section{Ethics}

All methods associated with animals described in this study were conducted in accordance with the Institutional Animal Care and Research Committee (IACUC) approved protocols at the University of Nebraska-Lincoln (UNL) and University of Nebraska Medical Center (UNMC).

\section{hIL34-Tg NOG and NOG Mice}

NOG (NOD.Cg-Prkdc $c^{\text {scid }}$ Il2 gg $^{\text {tm1Sug} / J i c T a c) ~ m i c e ~ o f ~ 6-8-w e e k-~}$ old were purchased from Taconic Biosciences (Rensselaer, NY 12144, United States) and housed at the UNL Life Sciences Annex under specific-pathogen-free conditions. The hIL34-Tg NOG mice were bred at UNMC by pairing hIL34-Tg mouse with NOG mouse of opposite gender. The offspring were genotyped after three weeks of age by obtaining DNA from the tail snipping. Genotyping was performed for hIL34 as described previously using real-time polymerase chain reaction (19). Mice positive for hIL34 were transferred to UNL to generate hu-BLT mice.

\section{Generation of hu-BLT-hIL34 and hu-BLT Mice}

To investigate human microglia reconstitution in the CNS of huBLT-hIL34 mice, both hu-BLT-hIL34 and hu-BLT mice were generated from adult hIL34-Tg NOG and NOG mice as we previously reported $(22,23)$ (Figure 1A). Briefly, 6-to-8-week-old adult hIL34-Tg NOG mice and NOG mice received sublethal irradiation at the dose of $12 \mathrm{cGy} / \mathrm{gram}$ of body weight with an RS2000 X-ray irradiator (Rad Source Technologies). Mice were surgically engrafted with a sandwich of two pieces of human fetal liver and one piece of thymic tissue fragments under the murine left renal capsules, of which human fetal livers and thymus tissues were procured from the Advanced Bioscience Resources. Within 6 hours after surgery, a total of $2.3 \times 10^{5}$ fetal liver derived CD $34^{+}$HSPCs in $200 \mathrm{ul}$ volume was injected through tail vein. At 16 weeks post engraftment, the immune reconstitution in the peripheral blood was assessed using flow cytometry as described below.

\section{Human Brain Tissues}

To compare the human microglia from hu-BLT-hIL34 mice with humans, ethically sourced human autopsy cerebral cortex tissues from a deidentified individual of $\mathrm{HIV}-1$ negative with no registered medical complications were obtained from the NIH Neuro BioBank (https://neurobiobank.nih.gov/).

\section{Multicolor Flow Cytometry}

Multicolor flow cytometry was conducted as previously reported $(22,23)$ for assessing the human immune reconstitution of peripheral blood mononuclear cells (PBMCs) at 16- and 20weeks post transplantation (Figure 1B). Briefly, peripheral blood was collected from a great saphenous vein into the ethylenediaminetetraacetic acid (EDTA)-containing vial (BD Microtainer, Franklin Lakes, NJ, USA). Red blood cells were lysed using fluorescence activated cell sorting (FACS) lysing solution (BD Biosciences, USA). The PBMCs were resuspended in a FACS buffer ( $2 \%$ FBS in phosphate-buffered saline) and incubated with a cocktail of following monoclonal antibodies against human immune cell markers at $4^{\circ} \mathrm{C}$ for $30 \mathrm{~min}$ : antihCD45-fluorescein isothiocyanate (FITC), anti-hCD3Phycoerythrin (PE), anti-hCD19-Phycoerythrin-Cyanin 5 (PECy5), anti-hCD4-Alexa Fluor 700 (AF700), anti-hCD8allophycocyanin-Cyanin 7(APC-Cy7) and anti-hCD14-Brilliant Violet 605 (BV605). All above antibodies and isotype control 

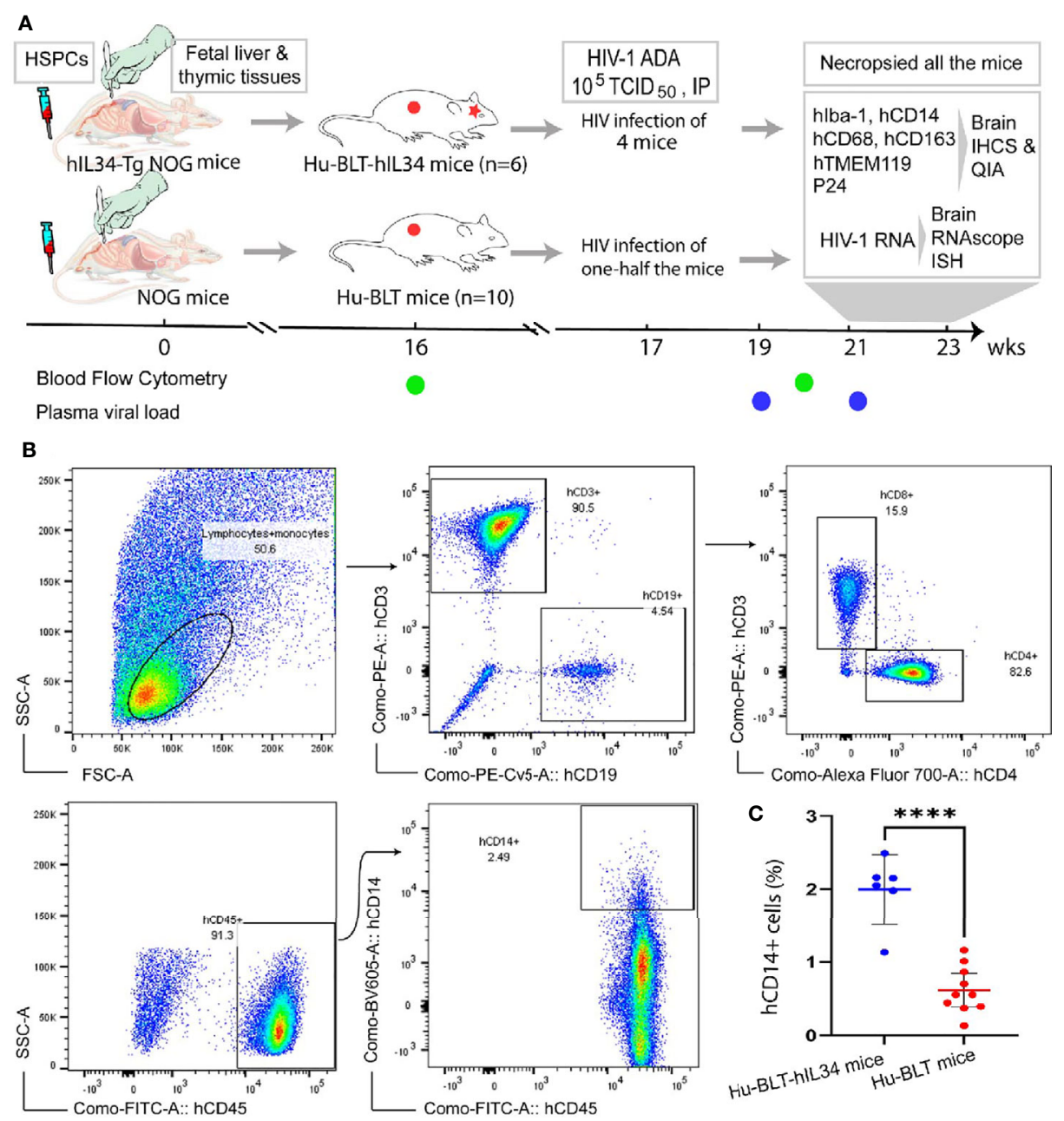

FIGURE 1 | Experimental timeline and Flow Cytometric evaluation of human immune cells reconstitutions in the peripheral blood of hu-BLT-hIL34 and hu-BLT mice. (A) Experimental design and timeline for the humanization of hlL34 transgenic mice and NOG mice by engrafting with human CD34+ hematopoietic stem and progenitor cells (HCSPs) and human fetal liver and thymic tissues, HIV-1 Ada infection of some mice in each group, and the evaluation of human myeloid and microglial cell reconstitution and HIV-1 infection in the CNS of euthanized mice using immunohistochemical staining (IHCS) and RNAscope in situ hybridization (ISH). (B) The representative flow cytometric dot plots of the peripheral blood mononuclear cells of a hu-BLT-hIL34 mouse (\#1703) at the 4 months after transplantation, which were gated with hCD45+, hCD3+, hCD9+, and hCD14+ cells. (C) The percentage of hCD14+ myeloid cells in the peripheral blood of hu-BLT-hIL34 mice is significantly higher than that hu-BLT mice at the 4 months post transplantation. Each symbol represents an individual mouse, hu-BLT-hIL34 mice n=6, hu-BLT mice $n=10 ;{ }^{\star \star \star \star} p<0.0001$.

antibodies were obtained from BD Biosciences, USA. The stained cells were washed with FACS buffer and fixed with $4 \%$ paraformaldehyde. Raw data were acquired by FACSAria III (BD Biosciences) and analyzed with FlowJo version 7.6.4 (TreeStar). Statistical analysis was performed using GraphPad Prism 7 (GraphPad Software). Two groups of female hu-BLThIL34 $(n=6)$ and hu-BLT mice $(n=10)$ with good human immune cell reconstitutions in the peripheral blood (Table 1) were selected for this study.

\section{HIV-1 Infection and Measurement of HIV-1 Plasma Viral Loads}

To investigate the infectivity of HIV-1 in parenchymal human microglial cells in the CNS, 4 mice (\# 1703, 1705, 1708 and 1709) of the hu-BLT-hIL34 mice group and 5 mice (\# 1720, 1723, 1724, 1726 , and 1728) in the hu-BLT mice group were randomly selected (Table 1) and intraperitoneally inoculated with the $10^{5}$ tissue culture infectious dose $50\left(\mathrm{TCID}_{50}\right)$ of macrophage-tropic HIV-1 ADA in 200 $\mu$ l volume (obtained through the NIH AIDS Reagent Program, Division of AIDS, NIAID, NIH: HIV-1 ADA Virus from Dr. Howard Gendelman). At 2- and 4-weeks post HIV-1 inoculation, HIV-1 plasma viral load (pVL) in copies/ml was determined by real-time RT-PCR using our previously published protocol (24). Briefly, viral RNA was extracted from the plasma using QIAamp ViralRNA minikit (Qiagen) as recommended by the manufacturer and quantified using C1000 ThermalCycler and the CFX96 Real-Time system (BioRad). For $20 \mu \mathrm{l}$ qRT-PCR, $5 \mu \mathrm{l}$ of extracted viral RNA, TaqMan 
TABLE 1 | The blood human immune cells reconstitutions of hu-BLT-hIL34 and hu-BLT mice.

\begin{tabular}{|c|c|c|c|c|c|c|c|}
\hline Group & Mouse ID & hCD45 (\%) & hCD3+ (\%) & hCD8+ (\%) & hCD4+ (\%) & hCD19+ (\%) & hCD14+ (\%) \\
\hline \multirow[t]{6}{*}{ Hu-BLT-hIL34 } & 1699 & 92.10 & 88.90 & 14.70 & 83.80 & 7.76 & 1.98 \\
\hline & 1703 & 91.30 & 90.50 & 15.90 & 82.60 & 4.54 & 2.49 \\
\hline & 1705 & 16.60 & 94.60 & 49.00 & 49.00 & 0.88 & 2.05 \\
\hline & 1707 & 85.80 & 99.60 & 10.80 & 88.40 & 0.02 & 1.13 \\
\hline & 1708 & 83.50 & 91.20 & 11.80 & 87.00 & 4.93 & 2.16 \\
\hline & 1709 & 7.62 & 87.80 & 55.00 & 41.00 & 5.79 & 2.15 \\
\hline Mean & & 62.82 & 92.10 & 26.20 & 71.97 & 3.99 & 1.99 \\
\hline SD & & 39.52 & 4.35 & 20.16 & 21.14 & 2.97 & 0.46 \\
\hline \multirow[t]{10}{*}{$\mathrm{Hu}-\mathrm{BLT}$} & 1717 & 83.80 & 22.40 & 47.70 & 49.50 & 67.80 & 1.16 \\
\hline & 1718 & 89.50 & 84.30 & 19.80 & 78.50 & 13.20 & 0.37 \\
\hline & 1720 & 42.90 & 94.70 & 20.80 & 78.20 & 3.14 & 0.44 \\
\hline & 1721 & 78.90 & 99.50 & 11.20 & 87.90 & 0.02 & 0.13 \\
\hline & 1722 & 56.30 & 23.90 & 46.60 & 51.40 & 69.70 & 0.70 \\
\hline & 1723 & 89.10 & 64.20 & 24.30 & 73.60 & 32.70 & 0.55 \\
\hline & 1724 & 70.30 & 97.60 & 15.50 & 83.80 & 1.24 & 0.86 \\
\hline & 1726 & 64.70 & 54.70 & 72.10 & 26.50 & 31.00 & 1.01 \\
\hline & 1728 & 94.30 & 81.40 & 18.20 & 80.10 & 15.50 & 0.39 \\
\hline & 1729 & 93.30 & 83.60 & 17.30 & 80.90 & 13.10 & 0.55 \\
\hline Mean & & 76.31 & 70.63 & 29.35 & 69.04 & 24.74 & 0.62 \\
\hline $\mathrm{SD}$ & & 17.32 & 28.71 & 19.56 & 19.81 & 25.74 & 0.32 \\
\hline
\end{tabular}

Fast Virus 1-Step master mix (Life Technologies) and following primers and probe combination (IDT, USA) were used: Forward Primer, GCCTCAATAAAGCTTGCCTTGA; Reverse Primer, G G G C G C C A C T G C T A G A GA ; Probe,/ 56 - F A M / CCAGAGTCA/ZEN/CACAACAGACGGGCACA/3IABkFQ/.

\section{Euthanasia of All the Mice for Evaluating Human Myeloid Cell Reconstitution and HIV-1 Infection in the CNS}

After two consecutive positive results of HIV-1 pVL at 2- and 4weeks post HIV-1 inoculation, which is equivalent to the $21-$ to 23-weeks post transplantation, all the mice, including HIV-1 infected and non-inoculated subgroups from the hu-BLT-hIL34 and hu-BLT mice groups, were euthanized for analyzing human myeloid cell reconstitutions and HIV-1 infections in the CNS (Figure 1A). Whole brain was dissected out during necropsy and sliced coronally into 5 parts at $4 \mathrm{~mm}$ interval using a young mouse brain slicer (Cat\# BSMYS001-1, Zivic Instruments, Pittsburgh, PA, USA). The brain tissues and other tissues including spinal cord, spleen, lymph node, jejunum and ileum were collected and fixed in SafeFix ${ }^{\text {TM }}$ II (Cat\# 042600, Fisher Scientific, USA) at room temperature for 6 hours and embedded in paraffin.

\section{Immunohistochemical Staining (IHCS)}

To evaluate the phenotype, morphology and distribution of the reconstituted human myeloid cells in the CNS, IHCS was conducted by following our previously published protocol with slight modifications (25). Briefly, antigen retrieval of $6-\mu \mathrm{m}$ thick tissue sections were performed in $0.1 \mathrm{mM}$ citrate buffer $(\mathrm{PH} 6.0)$ by heating at $98^{\circ} \mathrm{C}$ for 15 mins. The following primary antibodies were used for detecting macrophage/microglial cells: rabbit monoclonal-antibody ( $\mathrm{mAb}$ ) to human ionized calcium-binding adaptor molecule 1 (hIba-1, EPR6136-2 clone, Cat\# ab221933, 1:500; Abcam, USA), rabbit polyclonal antibody to human TMEM119-C-terminal (hTMEM119, Cat\# ab185333, 1:500;
Abcam, USA), rabbit mAb to human CD14 (hCD14, EPR3653 clone, Cat. \# 133335, 1:1000; Abcam, USA), mouse mAb to human CD68 (hCD68, PG-M1 clone, Cat\# MS-1808-S1, 1:100; Thermoscientific, USA), mouse mAb to human CD163 (hCD163, 10D6 clone, Cat\# NCL-L-CD163, 1:100; Leica Biosystems, USA). For HIV-1 detection, mouse mAb to HIV-1 gag p24 (Kal-1 clone, Cat\# M0857, 1:10, Dako, USA) was used. Mouse or rabbit IgG isotype control antibodies were used as negative control. Dako EnVision+ system-HRP labelled polymer anti-rabbit kit (Code K4002, Dako, USA) or anti-mouse kit (Code K4000, Dako, USA), and the Betazoid DAB Chromogen Kit (Cat\# BDB2004, BioCare Medical, USA) were used for signal detection and visualization. The cell nuclei were counterstained with Mayer's hematoxylin. The stained tissue sections after completion of IHCS were digitized with Aperio CS2 Scanscope and the quantitative image analysis of positive cells was conducted using a positive pixel count algorithm in Aperio's Spectrum Plus analysis program (version 9.1; Aperio ePathology Solutions) as previously reported (26).

\section{HIV-1 Viral RNA Detection Using RNAscope In Situ Hybridization (ISH)}

HIV-1 viral RNA (vRNA) in the brain tissues were detected using RNAscope ISH according to our previously published protocol (26). Briefly, HIV-1 antisense probes of RNAscope ${ }^{\circledR}$ ISH probe-V-HIV1-clade B (Cat\# 416111) and RNAscope ${ }^{\circledR} 2.5$ HD assay-Red kit were used. The RNAscope ${ }^{\circledR}$ probe-DapB (Cat\# 310043) was used as a negative control. All the reagents above were purchased from the Advanced Cell Diagnostics, Inc.

\section{Combined RNAscope ISH With IHCS}

To determine the cell types of HIV-1 vRNA+ cells in the CNS, a combined RNAscope ISH and IHCS method was used as reported (26). Briefly, after the completion of RNAscope ISH for HIV-1 vRNA and digitization of the whole tissue section, the slides were soaked in xylene overnight to remove the coverslip and the tissue 
section was rehydrated and subjected to IHCS using the rabbit mAb to hIba-1 (clone\# EPR6136-2, Cat\# ab221933, 1:500; Abcam, USA) as the primary antibody as described in the IHCS section above. Rabbit IgG isotype control antibody was used as negative control.

\section{RESULTS}

\section{Human Myeloid Cell Reconstitution in the CNS}

CNS contains four types of macrophages: parenchymal microglia and nonparenchymal perivascular macrophages (PVM), meningeal macrophages (MM) as well as choroid plexus macrophages $(2,8)$. First, we used antibodies to hIba-1, hCD14, hCD68 and hCD163, sensitive markers of macrophages and microglial cells (27-30), to evaluate human myeloid cells reconstitutions in the CNS of huBLT-hIL34 and hu-BLT mice. We examined human myeloid cells and microglial reconstitutions in the CNS of all six hu-BLT-hIL34 mice using IHCS with antibodies to hIBA-1, hCD14, hCD68, hCD163, and hTMEM119. To compare, we also evaluated the human myeloid cells and microglial reconstitution in $6 \mathrm{hu}$-BLT mice using IHCS with above antibodies. The frequency and distribution of human myeloid cells and microglial cells in CNS of these mice are highly consistent among these different antibodies. There were extensively reconstitutions of human myeloid cells in the CNS parenchyma of hu-BLT-hIL34 mice as indicated by hIba-1+ cells (Figure 2 and Supplementary Figure 1), hCD14+ cells (Figure 3 and Supplementary Figure 2), CD68+ cells
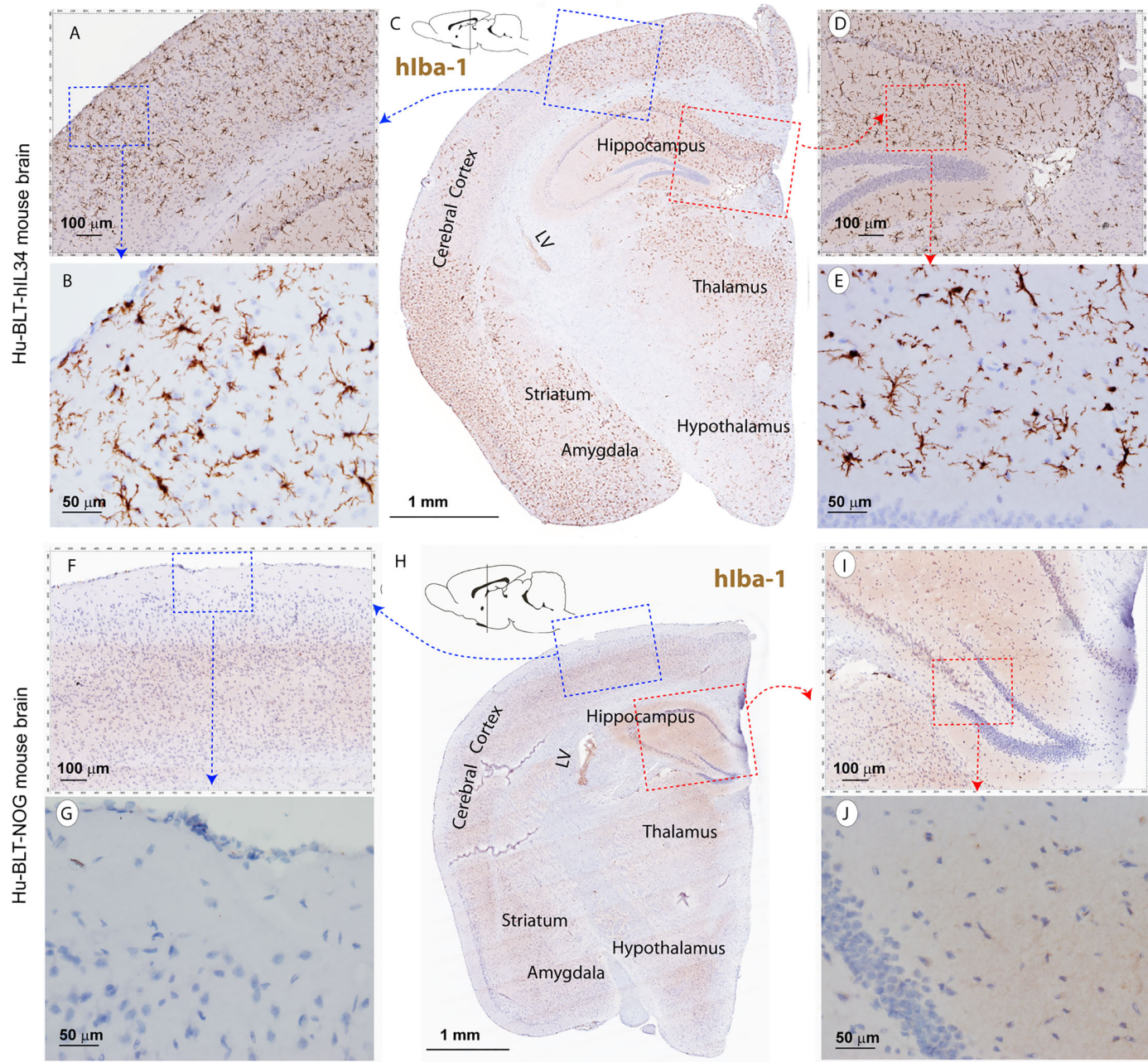

FIGURE 2 | The hlba-1+ myeloid cell reconstitutions in the brain tissues of hu-BLT-hlL-34 and hu-BLT mice. The upper panel (A-E) shows a representative whole brain tissue section from the third coronal brain slice of a hu-BLT-hlL34 mouse (\#1703) that was stained immunohistochemically for hlba-1+ (brown) and counterstained with hematoxylin. LV stands for lateral ventricle. The blue and red boxed regions in the Figure C were highlighted at a higher magnification (A, D). In turn, the blue and red boxed regions of the (A, B) were further highlighted (B, E). The hlba-1+ cells are morphologically ramified and mainly distributed in brain parenchyma. The lower panel (F-J) shows a representative whole brain tissue section (H) of a hu-BLT mouse (\#1717) that was stained immunohistochemically for hlba-1+ cells (brown). The blue and red boxed regions in the Figure $\mathrm{H}$ was highlighted at a higher magnification (F, I). In turn, the blue and red boxed regions of the (F, I) were further highlighted (G, J). There were no detectable parenchymal hlba-1+ cells. 

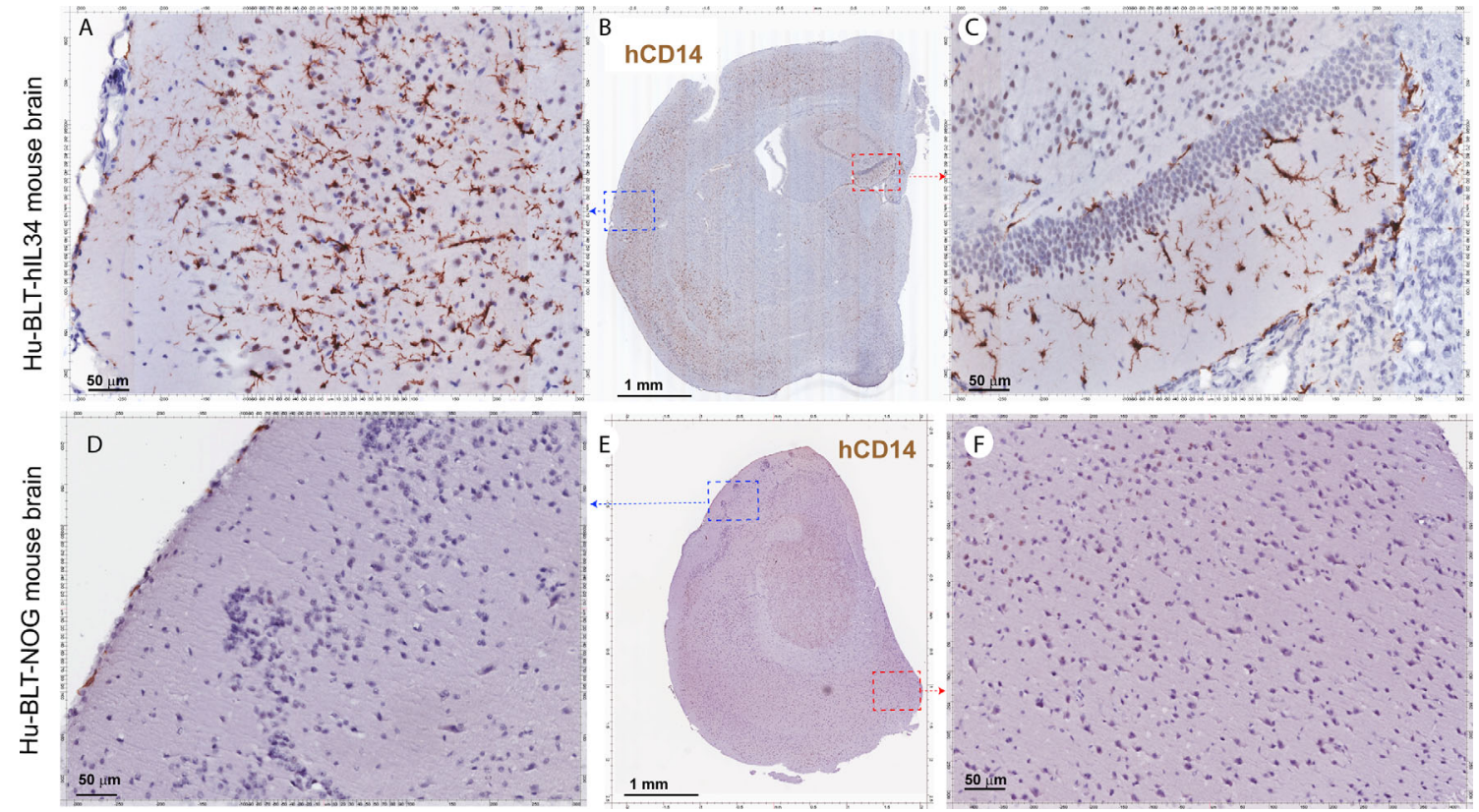

FIGURE 3 | The hCD14+ myeloid cell reconstitutions in the brain tissues of hu-BLT-hIL34 BLT and hu-BLT mice. The upper panel (A-C) shows representative whole brain tissue sections and the highlighted images form the corresponding boxed regions from hu-BLT-hIL34 mouse (\#1708) that were stained immunohistochemically for hCD14+ cells (brown) and counterstained with hematoxylin. There were abundant hCD14+ cells (A-C) in the brain of the hu-BLT-hlL34 mouse. The lower panel (D-F) in contrast shows no detectable hCD14+ myeloid cells in the brain parenchyma and a very limited number of hCD14+ cells in meninges in a representative whole brain tissue section of a hu-BLT mouse (\#1723).

(Supplementary Figure 2) and CD163+ cells (Supplementary Figure 3) using IHCS. As shown in a representative whole brain tissue section from the third coronary slice (Figure 2C) of all huBLT-hIL34 mouse (\#1703), hIba-1+ cells in the brain parenchyma were extensively reconstituted across multiple regions of the brain. The Figures 2A, D respectively highlighted the cerebral cortex and hippocampus boxed regions from the Figure 2C; in turn, Figures $\mathbf{2 B}$, E respectively further highlighted the boxed regions from the Figures 2A, D at a higher magnification. We also detected extensive reconstitution of hIba-1+ cells in spinal cord (Supplementary Figures 1A, B, mouse\# 1708) and cerebellum tissues (Supplementary Figures 1C, D, mouse\#1708) of hu-BLT-hIL34 mice. The hIba-1+ cells are numerous, morphologically ramified, distributed in brain parenchyma. Consistent with the extensive reconstitution of parenchymal human myeloid cells in the CNS revealed by hIba-1+ cells, there were also abundant hCD14+ myeloid cells as shown in a representative whole brain tissue sections (Figures 3A-C, mouse\#1708) of hu-BLT-hIL34 mice. The blue and red boxed regions of cerebral cortex and hippocampus in the Figure $\mathbf{3 B}$ were respectively highlighted at a higher magnification in the Figures 3A, C. The hCD14+ cells are again morphologically ramified and mainly distributed in brain parenchyma. Consistent with the extensive reconstitutions of hIBa$1+$ and hCD14+ cells above mentioned, there were also abundant hCD68+ cells (Supplementary Figure 2) and hCD163+ cells (Supplementary Figure 3) in the brain of hu-BLT-hIL34 mice. We thus concluded that hu-BLT-hIL34 mice extensively reconstituted parenchymal human myeloid cells in the CNS, including cerebral cortex, hippocampus, thalamus hypothalamus, striatum, amygdala, spinal cord, and cerebellum (Figures 2, 3 and Supplementary Figures 1-3). In contrast and as expected, there were an absence of detection or very limited reconstituted hIBa-1+ cells (Figures 2F-J, Supplementary Figures 1E-H), hCD14+ cells (Figures 3D-F and Supplementary Figure 2), hCD68+ cells (Supplementary Figure 2), or hCD163+ cells (Supplementary Figures 3D-F) cells in the CNS of the hu-BLT mice. As shown in a representative whole brain tissue section from the third coronal slice (Figures 2F-J, mouse\#1717) of hu-BLT mice, hIba-1+ cells were absent in all the parenchyma of cerebral brain (Figures $2 \mathbf{F}-\mathbf{J}$ ). Similarly, hCD14+ cells were also absent in the parenchyma of huBLT mice (Figures 3D-F, mouse\#1723). We next compare the reconstitution of nonparenchymal perivascular macrophages and meningeal macrophages in the hu-BLT-hIL34 and hu-BLT mice. As indicated by hIba-1, hCD14, hCD163 expression and their anatomic distribution, hu-BLT-hIL34 mice also have a better reconstitution of meningeal macrophages (MM) and perivascular macrophages (PVM) than hu-BLT mice (Figures 2, 3 and Supplementary Figures 1-3).

\section{The Myeloid Cells in Brain Parenchyma Expressed Microglia-Specific Marker hTMEM119}

To distinguish parenchymal microglia from macrophages, we conducted IHCS using microglial-specific marker hTMEM119 
$(31,32)$. There were extensively reconstitutions of hTMEM119+ cells in the CNS parenchyma of hu-BLT-hIL34 mice (Figures 4A-F, mouse\#1703). As shown in a representative whole brain tissue section from the third coronal slice (Figure 4C), hTMEM119+ cells in the brain parenchyma were extensively reconstituted across multiple regions of the brain. The Figures 4A, D respectively highlighted the boxed regions of the cerebral cortex and hippocampus from the Figure 4C; in turn Figures 4B, E respectively further highlighted the boxed regions from the
Figures 4A, D at a higher magnification. The hTMEM119+ cells are morphologically ramified and distributed in parenchyma. We quantified hTMEM119 + cells in the cerebral cortex of the hu-BTLhIL34 mice $(n=6)$ and found there were $304.08 \pm 131.93$ (mean \pm SD) hTMEM119+ microglial cells/mm2, whereas there were an absent of these cells in the hu-BLT mice. We further compared hTMEM119 + cells in the cerebral cortex of an HIV-1 non-infected individual, who had no registered medical complications at $\mathrm{NIH}$ Neuro Biobank. We found that the frequency, morphology, and
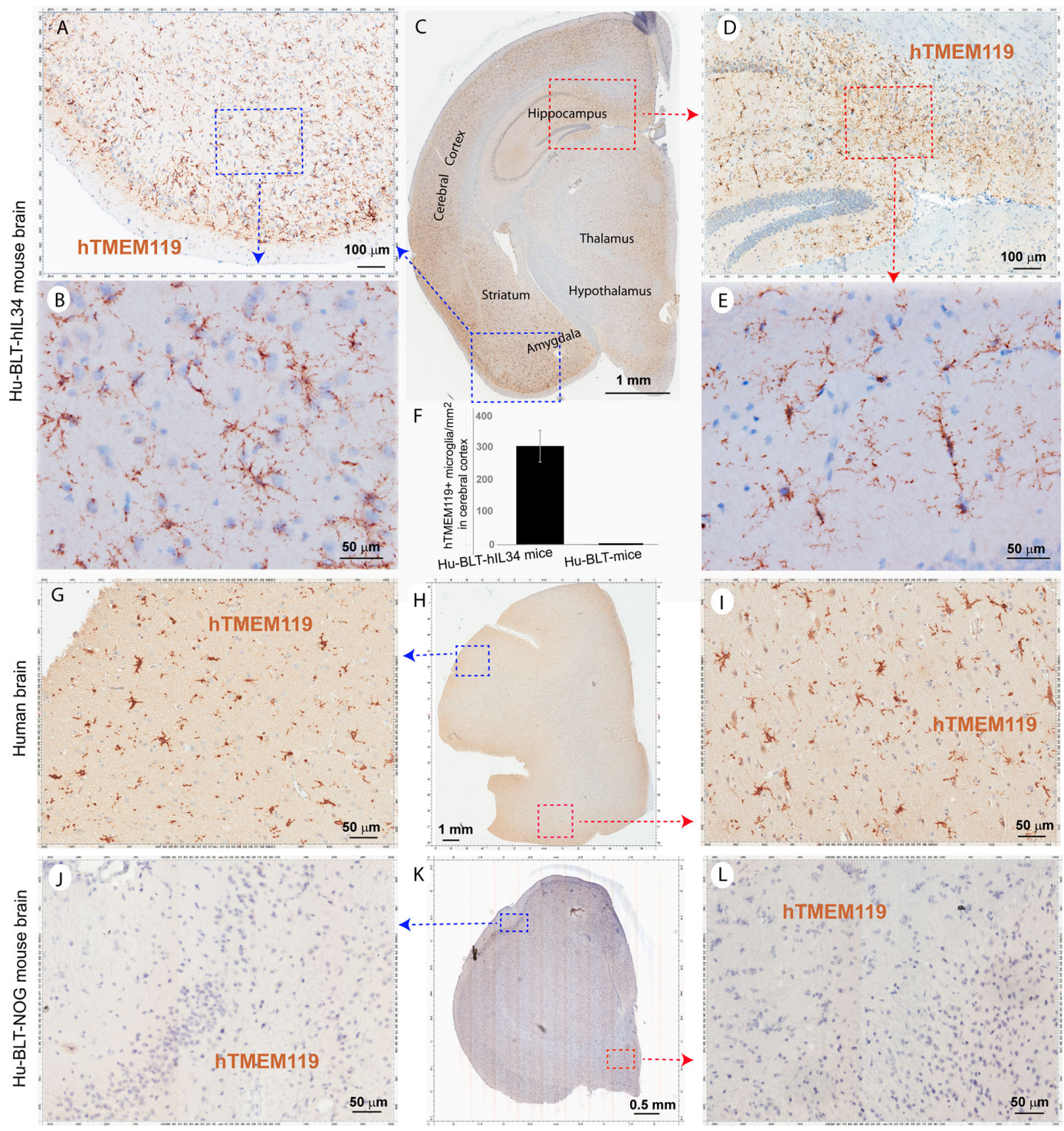

FIGURE 4 | The microglia-specific marker hTMEM119+ microglia in the brain tissues of hu-BLT-hlL34 and hu-BLT mice and humans. A representative whole brain tissue section (C) from the third coronal brain slice of a hu-BLT-hlL34 mouse (\#1703) that was stained immunohistochemically for hTMEM119+ cells (brown) and counterstained with hematoxylin. LV stands for lateral ventricle. The blue and red boxed regions in the Figure (C) were highlighted at a higher magnification (A, D). In turn, the blue and red boxed regions of the Figures (A, D) were highlighted (B, E). The hTMEME119+ cells (brown) are morphologically ramified and distributed in brain parenchyma. The histogram of quantitative images analysis of hTMEM119 + cells in the cerebral cortex of the hu-BTL-hlL34 mice (F) $(304.08 \pm 131.93$ hTEME119+ cells/mm2, n=6), where there were an absent of these cells in the hu-BLT mice. The middle panel (G-I) shows hTMEM119 + microglia in the cerebral cortex of a HIV-1 non-infected individual with no registered medical complications (G-I). The frequency, distribution, and morphology of hTMEM119+ human microglia in hu-BLT-hlL34 mice are similar to this human individual. The lower panel (J-L) shows no detectable hTMEM119+ cells in a representative whole brain section of a hu-BLT mouse (\#1721). 
distribution of hTMEM119+ cells in hu-BLT-hIL34 mice are similar to that person (Figures 4G-I). In contrast, there were an absence of detectable hTMEM119+ cells in the CNS of the hu-BLT mice (Figures 4J-L, mouse\#1721). We thus concluded that hu-BLThIL34 mice extensively reconstituted human microglia in the CNS.

\section{HIV-1 Infection in the CNS of hu-BLT-hIL34 Mice}

To test the functionality of reconstituted human myeloid cells in the CNS of hu-BLT-hIL34 mice, we infected 4 animals from huBLT-hIL34 group and 5 animals from hu-BLT mice groups (Supplemental Table 1). At 4-6 weeks post HIV-1 infection, we euthanized all 4 hu-BLT-hIL34 mice that were inoculated with HIV-1 Ada. We detected abundant HIV-1 vRNA+ and p24+ cells in the brain tissues of all 4 hu-BLT-hIL34 mice that were inoculated with HIV-1 (Figures 5A-D, mouse\#1708) but there were undetectable HIV-1 vRNA+ cells in three hu-BLT mice that were inoculated with $\mathrm{HIV}-1$ and examined (Figures 5E, F, mouse\#1724). As shown in representative images in the Figure 5, there were abundant HIV-1 RNA+ cells (red) in the brain tissues of hu-BLT-hIL34 mouse (\#1708) detected using RNAscope ISH with HIV-1 clade B probe. Consistent with the results of HIV-1 VRNA+ cells, there were abundant HIV-1 p24+ cells detected using IHCS. We further defined the HIV-1 vRNA+ cells type as human myeloid cells using IHCS with hIba-1 marker (Figure 5D, arrows) indicating the reconstituted human myeloid cells can support HIV-1 infection. In contrast, we did not detect any HIV-1 vRNA + cells in the brain of hu-BLT mice (Figures 5E, F, mouse\#1724).

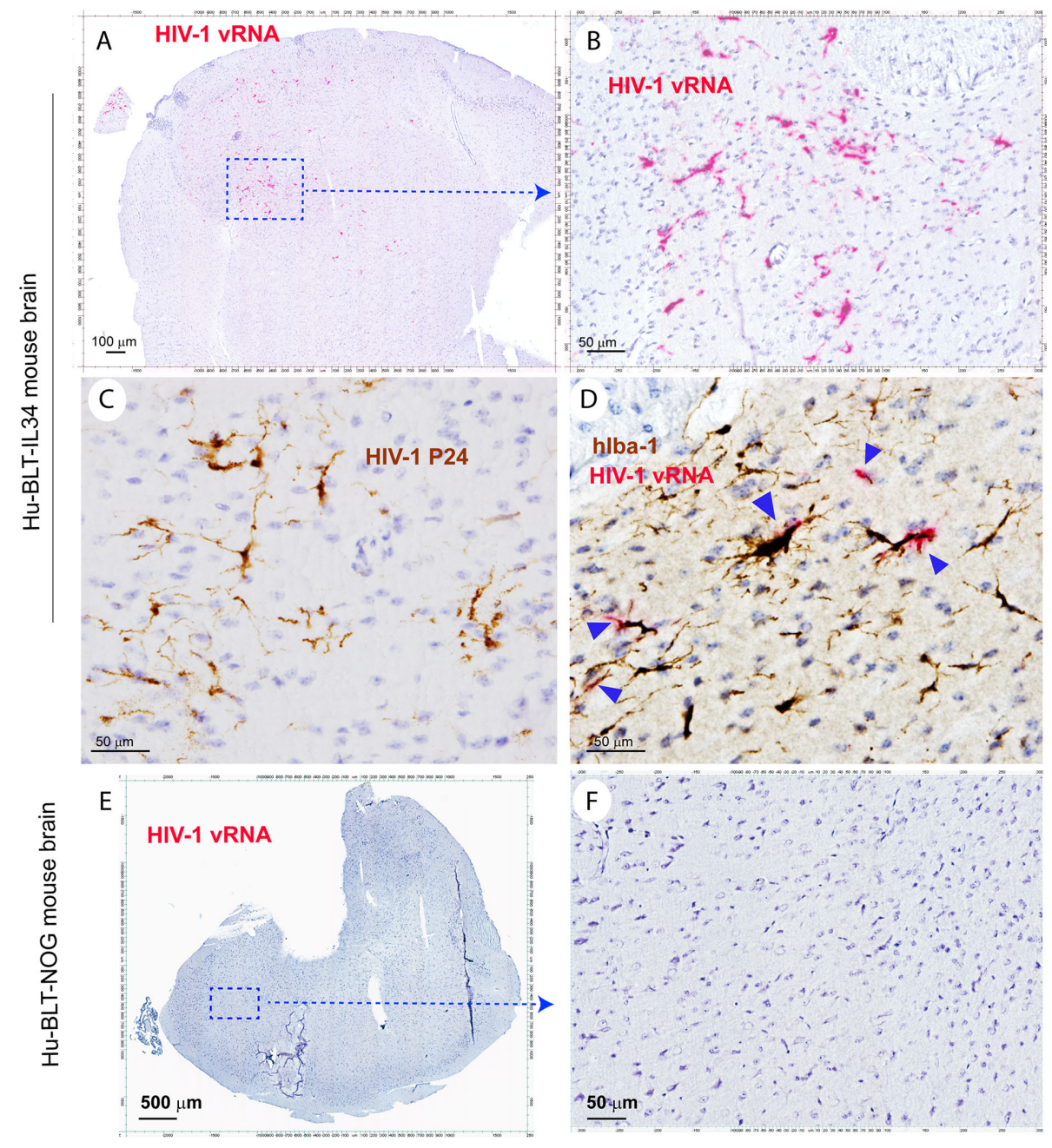

FIGURE 5 | The HIV-1 infection of human microglia of hu-BLT-hIL34 mice. The Upper panel shows HIV-1 RNA+ cells (A, B, red) in a representative whole brain tissue section from a hu-BLT-hlL34 mouse (\#1708) that was detected using RNAscope in situ hybridization with HIV-1 clade B anti-sense probe and counterstained with hematoxylin. The highlighted boxed region from the Figure (A) was shown at a higher magnification of HIV-1 RNA+ cells (B, red). HIV-1 p24+ cells (C, brown) in the cerebral cortex of the same mouse were detected using IHCS. The colocalization of HIV-1 VRNA and human myeloid cell marker hlba-1 (D, arrows) indicating the reconstituted human myeloid cells could support HIV-1 replication. The lower panel (E, F) shows there were no detectable HIV-1 RNA+ cells in a representative whole brain tissue section from a hu-BLT mouse (\#1724) using RNAscope in situ hybridization. 


\section{DISCUSSION}

In this study, we demonstrated that human microglia can be extensively reconstituted in CNS from circulating human HSPCs in hu-BLT-hIL34 mice. We first used a battery of human myeloid cell markers, including hIBa-1, hCD14, hCD68 and hCD163, to evaluate human myeloid cell reconstitution in the CNS and found that human myeloid cells were extensively reconstituted and primarily localized in the brain parenchyma (Figures 2, 3 and Supplementary Figures 1-3). We then used a human microglial specific marker, hTMEM119, to validate these reconstituted human myeloid cells in the brain parenchyma are mainly human microglia (Figures 4A-F). Further, in comparison with hTMEM119 + microglia in the cerebral cortex of a HIV-1 non-infected individual with no registered medical complications (Figures 4G-I), we found the frequency, distribution, and morphology of hTMEM119+ human microglia in hu-BLT-hIL34 mice are similar to this person. Our data thus support the notion that human microglia at adults can be generated through human hematopoietic stem and progenitor cells (HSPC), which is consistent with the previous reports that bone marrow derived cells can enter brain to different into microglia at adults (9-11). In contrast, we did not observe human microglia reconstitution in the brain parenchymal of hu-BLT mice (Figures 2-4 and Supplementary Figures 1-3 lower panels). The hu-BLT-hIL34 and hu-BLT mice are genetically identical and also received the same human donor transplant except the former had hIL34 knock-in, indicating that hIL34, a ligand of the colony stimulating factor-1 receptor, play an important role in myeloid and microglial cells development in CNS $(19,33)$. This study is unique in the following aspects. First human myeloid and microglia cells reconstitution in the CNS is a clear-cut result in this chimeric mouse and human model. Second, the adult mice engrafted with HSPCs extensively reconstituted human microglia in the CNS, to our knowledge this is the first report in this regard. In addition to comparing the parenchymal human microglia between hu-BLT-hIL34 and huBLT mice, we also observed that hu-BLT-hIL34 mice had a much better reconstitution of meningeal and perivascular macrophages than hu-BLT mice (Figures 2, 3 and Supplementary Figure 3). We also would like to point that both hu-BLT-hIL34 and hu-BLT mice received sublethal irradiation, whether this irradiation facilitated HSPCs to gain an entry into the brain and whether without irradiation can also reconstitute the human brain microglia in adult hIL34-NOG mice remains to be investigated.

Despite the importance of microglial cells, as a resident macrophage, in host immune response to brain infections and in the pathogenesis of neurodegenerative diseases, very limited small animal models are available to recapitulate diseases pathogenesis associated with human microglia. To that end, we infected hu-BLT-hIL34 mice and found that reconstituted human microglia are susceptible to HIV-1 infection in the CNS. Moreover, HIV-1 vRNA were localized in human myeloid cells in the brain, reenforcing that microglia cells are the most important subtract of HIV-1 infection in the CNS (26, 34). Using hu-BLT-hIL34 mice, it is now feasible to investigate the interplay between human pathogens, such as HIV-1, with human immune system of periphery and CNS compartments. The hu-BLT-hIL34 mouse model reported here open a new avenue for investigating the pathogenesis of HIV-1 infection and purging $\mathrm{HIV}-1$ latent reservoir in the CNS in addition to peripheral tissues.

\section{DATA AVAILABILITY STATEMENT}

The original contributions presented in the study are included in the article/Supplementary Material. Further inquiries can be directed to the corresponding authors.

\section{ETHICS STATEMENT}

Ethically sourced human autopsy cerebral cortex tissues from a deidentified individual of HIV-1 negative with no registered medical complications were obtained from the NIH Neuro BioBank (https://neurobiobank.nih.gov/). The patients/ participants provided their written informed consent to participate in this study. All methods associated with animals described in this study were conducted in accordance with the Institutional Animal Care and Research Committee (IACUC) approved protocols at the University of Nebraska-Lincoln (UNL) and University of Nebraska Medical Center (UNMC).

\section{AUTHOR CONTRIBUTION}

QL and JZ designed the experiments and wrote the manuscript. SG and LG bred the mice. JZ, SL, and YC generated hu-BLT mice and conducted animal HIV-1 infection experiment. JZ performed immunohistochemical staining and in situ hybridization on animal tissues and W-KK did the human brain tissue immunohistochemical staining. TW did the quantification of hTMEM119 + cells. All authors contributed to the article and approved the submitted version.

\section{FUNDING}

This study is supported in part by the National Institutes of Health (NIH) Grants P30 MH062261-16A1 Chronic HIV Infection and Aging in NeuroAIDS (CHAIN) Center (to Buch \& Fox), R21 AI143405 (to QL). R01 AI136756 (YL, QL). The funders had no role in study design, data collection and analysis, preparation of the manuscript, or decision for publication.

\section{ACKNOWLEDGMENTS}

We would like to thank University of Nebraska-Lincoln Life Sciences Annex and their staff for their assistance. 


\section{SUPPLEMENTARY MATERIAL}

The Supplementary Material for this article can be found online at: https://www.frontiersin.org/articles/10.3389/fimmu.2021.672415/ full\#supplementary-material

Supplementary Figure 1 | The hlba-1+ myeloid cell reconstitutions in the spinal cord and cerebellum tissues of hu-BLT-hIL-34 and hu-BLT mice. The upper panel shows abundant hlba-1+ cells (brown) from representative spinal cord (A, B, mouse\# 1708) and cerebellum (C, D, mouse\# 1703) tissue sections of a hu-BLThIL34 mice detected using IHCS. The lower panel shows no detectable hlba-1+ cells (brown) from representative spinal cord (E, F, mouse\# 1721) and cerebellum (G, H, mouse\#1717) tissue sections of a hu-BLT mice detected using IHCS.

Supplementary Figure 2 | The comparison of human myeloid cell reconstitutions in the cortex, corpus striatum, hippocampus, and cerebellum

\section{REFERENCES}

1. Kettenmann H, Hanisch U-K, Noda M, Verkhratsky A. Physiology of Microglia. Physiol Rev (2011) 91:461-553. doi: 10.1152/physrev. 00011.2010

2. Fani Maleki A, Rivest S. Innate Immune Cells: Monocytes, Monocyte-Derived Macrophages and Microglia as Therapeutic Targets for Alzheimer's Disease and Multiple Sclerosis. Front Cell Neurosci (2019) 13:355. doi: 10.3389/ fncel.2019.00355

3. Li Q, Barres BA. Microglia and Macrophages in Brain Homeostasis and Disease. Nat Rev Immunol (2018) 18:225-42. doi: 10.1038/nri.2017.125

4. Kierdorf K, Erny D, Goldmann T, Sander V, Schulz C, Perdiguero EG, et al. Microglia Emerge From Erythromyeloid Precursors Via Pu.1- and Irf8dependent Pathways. Nat Neurosci (2013) 16:273-80. doi: 10.1038/nn.3318

5. Alliot F, Godin I, Pessac B. Microglia Derive From Progenitors, Originating From the Yolk Sac, and Which Proliferate in the Brain. Dev Brain Res (1999) 117:145-52. doi: 10.1016/S0165-3806(99)00113-3

6. Ginhoux F, Lim S, Hoeffel G, Low D, Huber T. Origin and Differentiation of Microglia. Front Cell Neurosci (2013) 7:45. doi: 10.3389/fncel.2013.00045

7. Ginhoux F, Greter M, Leboeuf M, Nandi S, See P, Gokhan S, et al. Fate Mapping Analysis Reveals That Adult Microglia Derive From Primitive Macrophages. Science (2010) 330:841-5. doi: 10.1126/science.1194637

8. Goldmann T, Wieghofer P, Jordão MJC, Prutek F, Hagemeyer N, Frenzel K, et al. Origin, Fate and Dynamics of Macrophages at Central Nervous System Interfaces. Nat Immunol (2016) 17:797-805. doi: 10.1038/ni.3423

9. Simard AR, Soulet D, Gowing G, Julien JP, Rivest S. Bone Marrow-Derived Microglia Play a Critical Role in Restricting Senile Plaque Formation in Alzheimer's Disease. Neuron (2006) 49:489-502. doi: 10.1016/j.neuron. 2006.01.022

10. Mildner A, Schmidt H, Nitsche M, Merkler D, Hanisch U-K, Mack M, et al. Microglia in the Adult Brain Arise From Ly-6ChiCCR2+ Monocytes Only Under Defined Host Conditions. Nat Neurosci (2007) 10:1544-53. doi: $10.1038 / \mathrm{nn} 2015$

11. Hess DC, Abe T, Hill WD, Studdard AM, Carothers J, Masuya M, et al. Hematopoietic Origin of Microglial and Perivascular Cells in Brain. Exp Neurol (2004) 186:134-44. doi: 10.1016/j.expneurol.2003.11.005

12. Shultz LD, Brehm MA, Garcia-Martinez JV, Greiner DL. Humanized Mice for Immune System Investigation: Progress, Promise and Challenges. Nat Rev Immunol (2012) 12:786-98. doi: 10.1038/nri3311

13. Ito R, Takahashi T, Katano I, Ito M. Current Advances in Humanized Mouse Models. Cell Mol Immunol (2012) 9:208-14. doi: 10.1038/cmi.2012.2

14. Allen TM, Brehm MA, Bridges S, Ferguson S, Kumar P, Mirochnitchenko O, et al. Humanized Immune System Mouse Models: Progress, Challenges and Opportunities. Nat Immunol (2019) 20:770-4. doi: 10.1038/s41590-019-0416-Z

15. Marsden MD, Zack JA. Studies of Retroviral Infection in Humanized Mice. Virology (2015) 479-480:297-309. doi: 10.1016/j.virol.2015.01.017

16. Gorantla S, Makarov E, Finke-Dwyer J, Castanedo A, Holguin A, Gebhart CL, et al. Links Between Progressive Hiv-1 Infection of Humanized Mice and Viral Neuropathogenesis. Am J Pathol (2010) 177:2938-49. doi: 10.2353/ajpath. 2010.100536 tissues of hu-BLT-hIL-34 and hu-BLT mice. Every upper panel respectively shows human myeloid lineage cells of hlba-1+, hCD14+, hCD68+ or hTMEM119+ were extensively reconstituted throughout the cortex, corpus striatum, hippocampus and cerebellum of brains of hu-BLT-hIL34 mice; while every lower panel respectively shows an absence of or limited detection of these cells from hu-BLT mice using IHCS. Each amplified micrograph was shown from the red frame in the left upper corner thumbnails pictures. Scale bar equals $25 \mu \mathrm{m}$.

Supplementary Figure 3 | The comparison of human meningeal macrophages (MM) and perivascular macrophages (PVM) reconstitutions in the brain tissues of hu-BLT-hlL-34 and hu-BLT mice. The upper panel shows abundant hCD163+ (brown) human parenchymal myeloid cells (A-C), human meningeal macrophages (A, arrows) and human perivascular macrophages (C) from representative brain tissue sections of a hu-BLT-hlL34 mice (\#1708) detected using IHCS. The lower panel shows an absence of hCd163+ human parenchymal myeloid cells and a few of human meningeal macrophages (D-F, arrows) of hu-BLT mice (mouse\#1726).

17. Gorantla S, Poluektova L, Gendelman HE. Rodent Models for HIV-associated Neurocognitive Disorders. Trends Neurosci (2012) 35:197-208. doi: 10.1016/ j.tins.2011.12.006

18. Honeycutt JB, Liao B, Nixon CC, Cleary RA, Thayer WO, Birath SL, et al. T Cells Establish and Maintain CNS Viral Infection in HIV-infected Humanized Mice. J Clin Invest (2018) 128:2862-76. doi: 10.1172/JCI98968

19. Mathews S, Branch Woods A, Katano I, Makarov E, Thomas MB, Gendelman HE, et al. Human Interleukin-34 Facilitates Microglia-Like Cell Differentiation and Persistent HIV-1 Infection in Humanized Mice. Mol Neurodegener (2019) 14:12-2. doi: 10.1186/s13024-019-0311-y

20. Melkus MW, Estes JD, Padgett-Thomas A, Gatlin J, Denton PW, Othieno FA, et al. Humanized Mice Mount Specific Adaptive and Innate Immune Responses to EBV and TSST-1. Nat Med (2006) 12:1316-22. doi: 10.1038/nm1431

21. Lan P, Tonomura N, Shimizu A, Wang S, Yang YG. Reconstitution of a Functional Human Immune System in Immunodeficient Mice Through Combined Human Fetal Thymus/Liver and CD34+ Cell Transplantation. Blood (2006) 108:487-92. doi: 10.1182/blood-2005-11-4388

22. Sun M, Li Y, Yuan Z, Lu W, Kang G, Fan W, et al. VRC01 Antibody Protects Against Vaginal and Rectal Transmission of Human Immunodeficiency Virus 1 in hu-BLT Mice. Arch Virol (2016) 161:2449-55. doi: 10.1007/s00705-016-2942-4

23. Li Q, Tso FY, Kang G, Lu W, Li Y, Fan W, et al. Early Initiation of Antiretroviral Therapy Can Functionally Control Productive HIV-1 Infection in Humanized-BLT Mice. J Acquir Immune Defic Syndr (2015) 69:519-27. doi: 10.1097/QAI.0000000000000687

24. Yuan Z, Kang G, Ma F, Lu W, Fan W, Fennessey CM, et al. Recapitulating CrossSpecies Transmission of Simian Immunodeficiency Virus Sivcpz to Humans by Using Humanized BLT Mice. J Virol (2016) 90:7728-39. doi: 10.1128/JVI.00860-16

25. Li Q, Duan L, Estes JD, Ma Z-M, Rourke T, Wang Y, et al. Peak SIV Replication in Resting Memory CD4+ T Cells Depletes Gut Lamina Propria CD4+ T Cells. Nature (2005) 434:1148-52. doi: 10.1038/nature03513

26. Ko A, Kang G, Hattler JB, Galadima HI, Zhang J, Li Q, et al. Macrophages But Not Astrocytes Harbor HIV DNA in the Brains of HIV-1-Infected Aviremic Individuals on Suppressive Antiretroviral Therapy. J Neuroimmune Pharmacol (2019) 14:110-9. doi: 10.1007/s11481-018-9809-2

27. Janova H, Böttcher C, Holtman IR, Regen T, van Rossum D, Götz A, et al. CD14 is a Key Organizer of Microglial Responses to CNS Infection and Injury. Glia (2016) 64:635-49. doi: 10.1002/glia.22955

28. Beschorner R, Nguyen TD, Gözalan F, Pedal I, Mattern R, Schluesener HJ, et al. CD14 Expression by Activated Parenchymal Microglia/Macrophages and Infiltrating Monocytes Following Human Traumatic Brain Injury. Acta Neuropathol (2002) 103:541-9. doi: 10.1007/s00401-001-0503-7

29. Walker DG, Lue L-F. Immune Phenotypes of Microglia in Human Neurodegenerative Disease: Challenges to Detecting Microglial Polarization in Human Brains. Alzheimer's Res Ther (2015) 7:56. doi: 10.1186/s13195-015-0139-9

30. Cosenza AM, Meng-Liang Z, Qiusheng SI, Lee SC. Human Brain Parenchymal Microglia Express CD14 and CD45 and are Productively Infected by HIV-1 in HIV-1 Encephalitis. Brain Pathol (2002) 12:442-55. doi: $10.1111 / j .1750-3639.2002 . t b 00461 . x$

31. Bennett ML, Bennett FC, Liddelow SA, Ajami B, Zamanian JL, Fernhoff NB, et al. New Tools for Studying Microglia in the Mouse and 
Human CNS. Proc Natl Acad Sci (2016) 113:E1738-46. doi: 10.1073/pnas. 1525528113

32. Satoh J-I, Kino Y, Asahina N, Takitani M, Miyoshi J, Ishida T, et al. TMEM119 Marks a Subset of Microglia in the Human Brain. Neuropathology (2016) 36:39-49. doi: 10.1111/neup.12235

33. Lelios I, Cansever D, Utz SG, Mildenberger W, Stifter SA, Greter M. Emerging Roles of IL-34 in Health and Disease. J Exp Med (2020) 217(3):e20190290. doi: 10.1084/jem.20190290

34. Wallet C, De Rovere M, Van Assche J, Daouad F, De Wit S, Gautier V, et al. Microglial Cells: The Main Hiv-1 Reservoir in the Brain. Front Cell Infect Microbiol (2019) 9:362. doi: 10.3389/fcimb.2019.00362
Conflict of Interest: The authors declare that the research was conducted in the absence of any commercial or financial relationships that could be construed as a potential conflict of interest.

Copyright $\odot 2021$ Zhang, Lohani, Cheng, Wang, Guo, Kim, Gorantla and Li. This is an open-access article distributed under the terms of the Creative Commons Attribution License (CC BY). The use, distribution or reproduction in other forums is permitted, provided the original author(s) and the copyright owner(s) are credited and that the original publication in this journal is cited, in accordance with accepted academic practice. No use, distribution or reproduction is permitted which does not comply with these terms. 